\title{
Location Based Group Selection in Resource Allocation for D2D Uplink Cellular Networks
}

\author{
Soo Hyeong Kang ${ }^{1}$, Bang Won $\mathrm{Seo}^{2}$ and ${ }^{*} J$ Jeong Gon Kim ${ }^{3}$ \\ ${ }^{1,3}$ Department of Electronic Engineering, Korea Polytechnic University \\ Siheung Si, Kyunggi Do, Korea 429-793 \\ ${ }^{2}$ Division of Electrical, Electronic \& Control Engineering, Kongju National \\ University \\ Cheonan, Republic of Korea \\ e-mail :soo7586@naver.com ${ }^{1}$,seobw@kongju.ac.kr ${ }^{2},{ }^{*} j g k i m @ k p u . a c . k r^{3}$
}

\begin{abstract}
Recently, mobile internet traffic has rapidly increased as the huge increase of the mobile devices and smart phone. If mobile internet traffic increases steadily, the resource for cellular network is not enough for the demand of huge internet traffic. Hence, D2D (Device-to-Device) technology is highlighted in order to resolve the lack of cellular resources and the demand of internet traffic. D2D communications use the same resource, which means that it shares the resource of cellular networks. But, D2D technology has some problems such as the mutual interference with celluar users by sharing same resource with cellular network and increased complexity to search the optimum resource for D2D links with minimizing the mutual interference with cellular users using same resources. In this paper, we proposed a efficient resource allocation for D2D links by using the location information for Cellular and D2D users, respectively. Proposed scheme utilizes location information and SINR (Signal-to-Interference-plusNoise Ratio) information in order to increase the efficiency of resource and also reduce computational complexity. Of the proposed scheme first selects the prospective cellular resources based on the location information and then finally D2D resource by SINR comparison from the group of selected resources in previous procedure. Simulation results show that the proposed scheme attains the higher throughput over the scheme with only location information and we also compare the D2D and cellular throughput of the proposed scheme according to the rate of the group selection for the next SINR comparison in order to determine the optimum resource for D2D communication.
\end{abstract}

Keywords: D2D communication, SINR, Location information, Cellular network, Uplink

\section{Introduction}

Recently, mobile internet traffic has rapidly increased as the huge increase of the smart phone and mobile devices. And along with the rapid development of wireless communication, the demands for higher data rate and better performance of the whole network become more and more important, which have promoted the research enthusiasm on the improvement of spectral efficiency and effective interference management [1]. D2D communication is a service for the direct communication between devices without passing through the eNB. It is possible to reduce the data traffic and to use limited frequency resource efficiently because D2D communication shares resources of cellular network. D2D communication resolve the problem of continually increasing data traffic and provide the proximity-based commercial services such as advertising or SNS [2]. For

${ }^{*}$ Corresponding Author 
example, multiple UEs with similar social interests may have a high chance of broadcasting similar contents (e.g., sharing the same video or audio clips) throughout networks. The transmission efficiency of the communication network can be further improved by combining these similar contents shared among groups of UEs with the same social interests, as shown in Figure 1 [3].

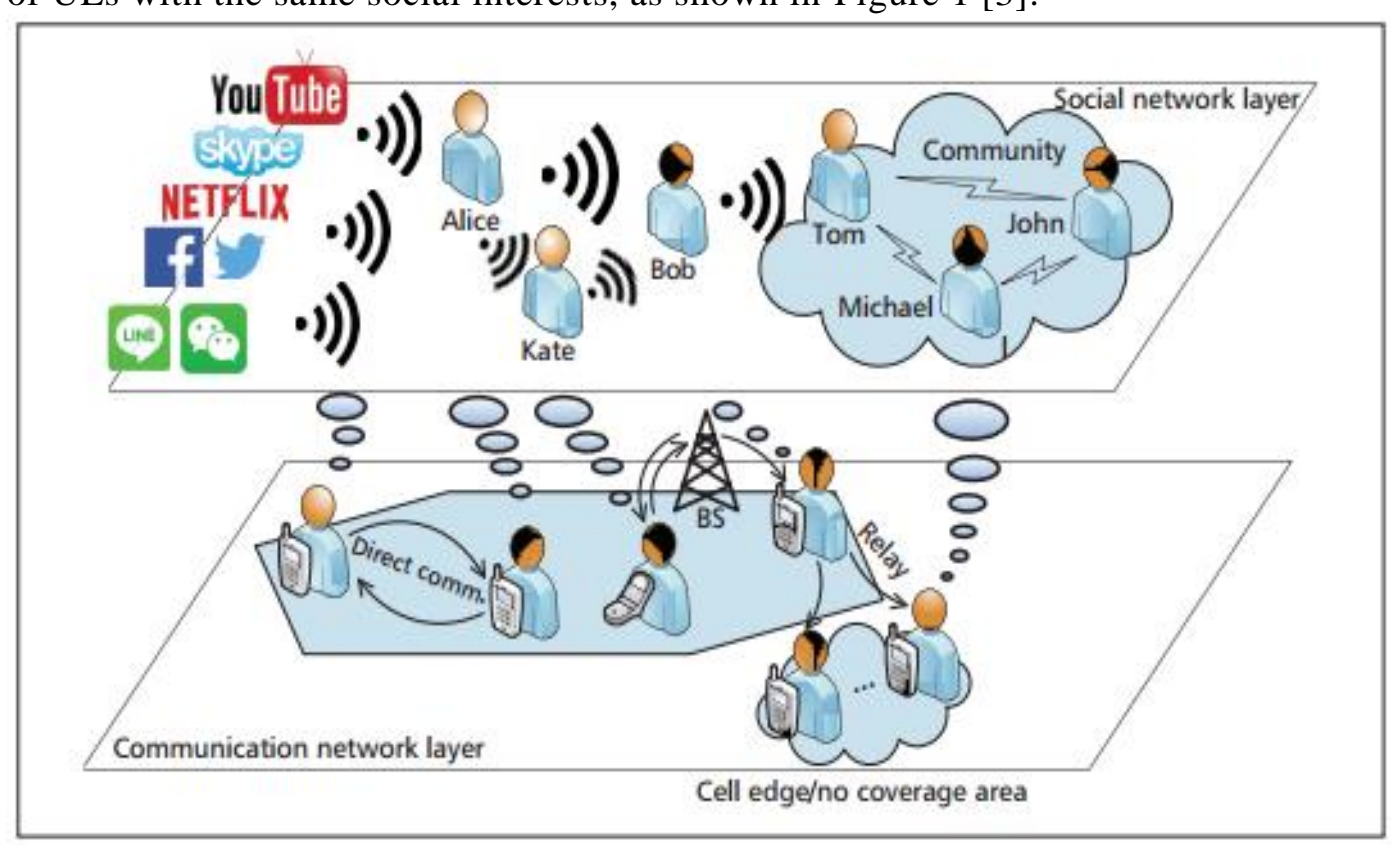

Figure 1. Social Network Enhanced Communication Networks

D2D communication technology in cellular networks was proceeded for standardization as entitled ProSe (Proximity Services) in $3 \mathrm{GPP}$ ( $3^{\text {rd }}$ Generation Partnership Project) Release 12 since 2011. 3GPP Release 13 is currently proceeding for standardization called eProSe (Enhancements to ProSe) that contain relay technology of D2D device [4-5].

D2D communications infrastructure has been proposed to take advantages of the physical proximity of communicating devices, increasing resource utilization, and improving cellular coverage [6]. However, D2D communication has some problems. D2D communication causes interference by using same resource with cellular user and D2D link. And increase computational complexity in order to allocate resource efficiently when D2D link use same resource with cellular network.

Currently, many researches on D2D communication have been focused on allocating resources and power control. There is a fixed dividing scheme for the available resource for cellular communication and for D2D communication. But, if there are relatively fewer D2D Pairs, it has the possibility to waste resources for full utilization [7]. Besides, there are also schemes that ensure performance by reducing the interference on the surrounding through power control [8]. However, those schemes consider the more parameter values and require the higher computational complexity. Some researchers have been studied on the use of power control to reduce the interference and computational complexity, while other approaches are relatively fewer than them of the power control based schemes since some years ago.

In this paper, we investigate the resource allocation schemes when the D2D links share the resources of the cellular network in the uplink. The proposed scheme utilizes the location information and SINR information. Location information is utilized in order to select some resources among all cellular resources. And SINR information is utilized in order to allocate resource among selected resources. Simulation results show proposed scheme is throughput and computational 
complexity as location only scheme and selected resource percent with $20 \%, 40 \%$, $60 \%, 80 \%$, and compared about the D2D link and CUE performance.

\section{System Model}

\subsection{System Model}

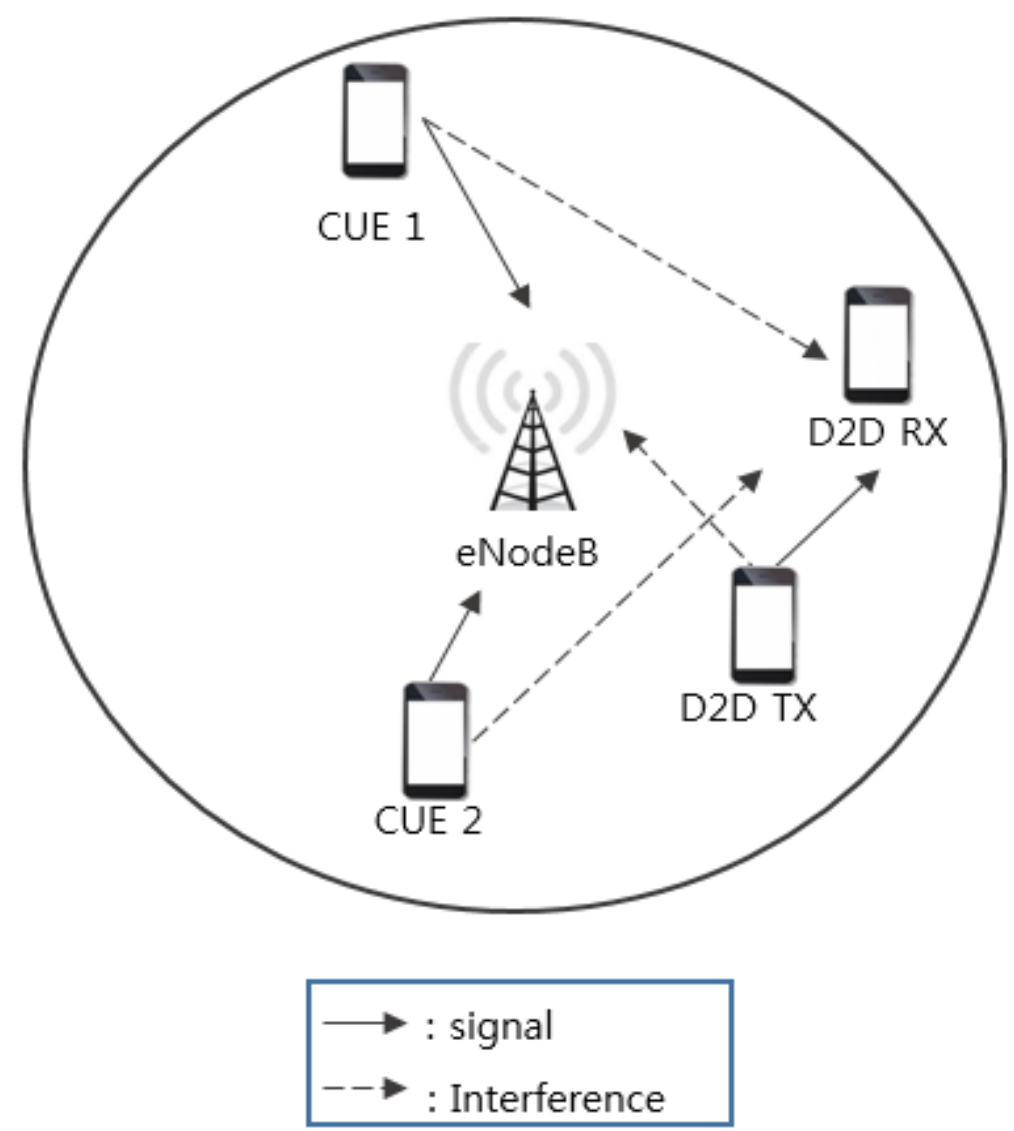

Figure 2. D2D Resource Allocation Scenarios in Cellular Network (Uplink)

In this paper, we consider the uplink scenario of single cellular. Cellular network is composed of the eNB, D2D link, CUE. The D2D link uses the same resources of the uplink cellular network. As shown in Figure 1, there is the interference scenario to share the same resource D2D link and CUE (Cellular User Equipment). The number of cellular network resource is same as the number of CUE. D2D link resources use the cellular network resource of one per D2D link of one.

The number of cellular network resource is $n$ and the number of CUE is also $n$ as the number of cellular network resource. But, the number of CUE is referred to $\mathrm{c}$ in order to separate the cellular network resources from CUE. The number of D2D link is d. We denote $N=\{1,2, \ldots, n\}$ as set of cellular network resource, $C=\{1,2, \ldots, c\}$ as set of CUE and D $=\{1,2, \ldots, d\}$ as set of D2D link.

It uses the symbol of $y_{c, d}$ to indicate the interference. In the case of $y_{c, d}=1$, it means that D2D link d share the resource of the CUE c. And in the case of $y_{c, d}=0$, it means that they do not share the resource.

SINR of CUE is thus defined as

$$
S I N R_{\text {Cellular }}=\frac{P_{\text {Cellular }} G_{c, e N B}}{P_{\text {noise }}+\sum_{d=1}^{D_{m} \text { max }} y_{c, d} P_{D 2 D} G_{d, e N B}}
$$


where $P_{\text {Cellular }}$ is the transmission power of the CUE, $G_{c, e N B}$ is the channel gain between CUE c and eNB and $P_{\text {noise }}$ is noise power. $P_{D 2 D}$ is the transmission power of the D2D link. $G_{d, e N B}$ is the channel hain between D2D link d and eNB. SINR of D2D link is thus defined as

$$
\operatorname{SINR}_{D 2 D}=\frac{P_{D 2 D} G_{D 2 D \text { Pair }}}{P_{\text {noise }}+\sum_{c=1}^{C_{m a x}} y_{c, d} P_{\text {Cellular }} G_{c, d}}
$$

where $G_{D 2 D \text { Pair }}$ is the channel gain between D2D link d TX and D2D link d RX and $G_{c, d}$ is the channel gain between CUE c and D2D link d.

The throughput can be determined by using (1) and (2).

$$
\begin{aligned}
& R_{\text {Cellular }}=\log _{2}\left(1+\operatorname{SINR}_{\text {Cellular }}\right) \\
& R_{D 2 D}=\log _{2}\left(1+\operatorname{SIN} R_{D 2 D}\right)
\end{aligned}
$$

where $R_{\text {Cellular }}$ represents the throughput rate of the CUE and $R_{D 2 D}$ represents the throughput rate of the D2D link.

\subsection{Location Based Resource Allocation}

Location Based Resource Allocation [9], which uses only the location information through GPS, determines resources to be shared with D2D link out of total available resources.

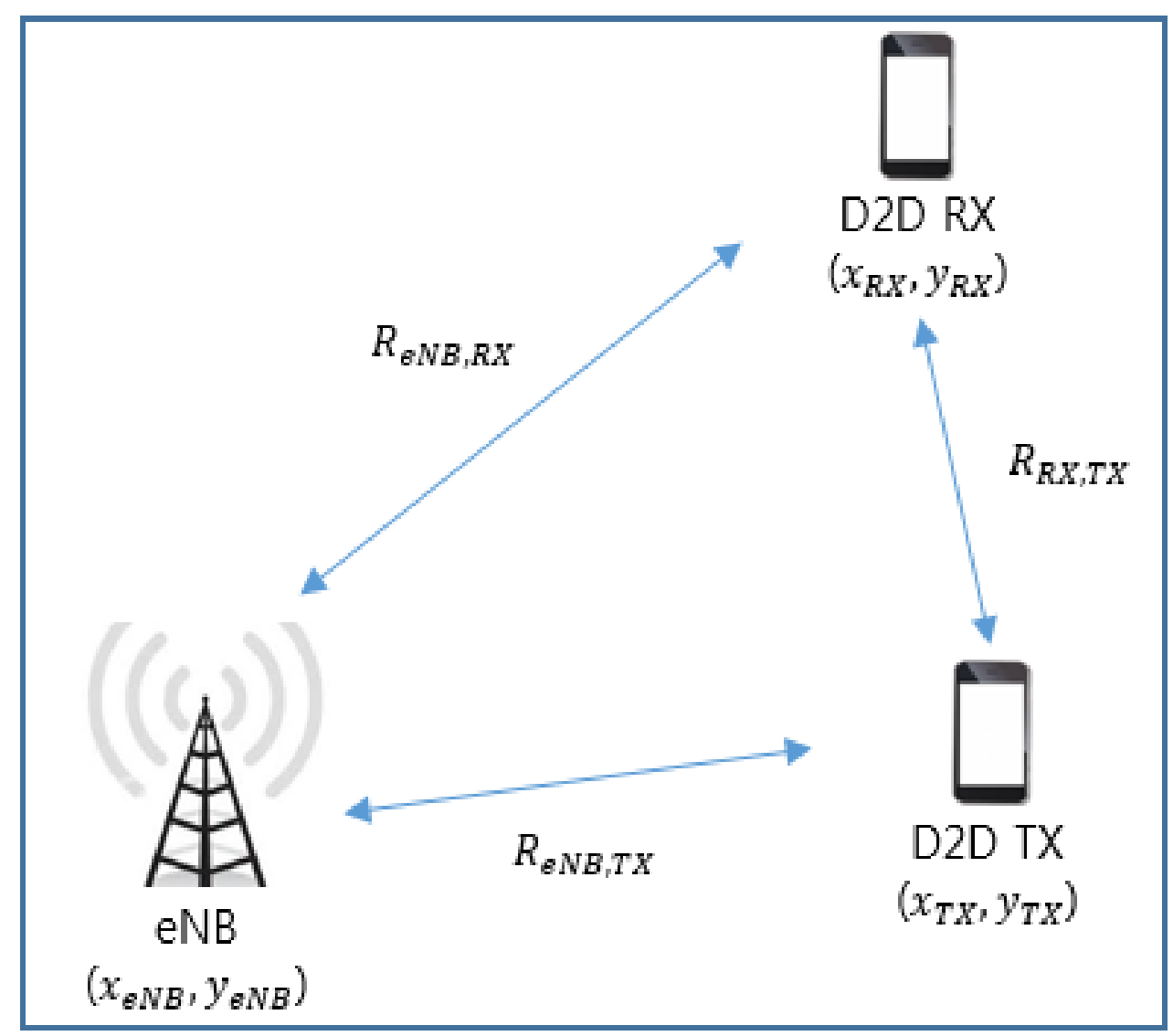

Figure 3. Location and Distance Information between eNB and D2D Links

As shown in Figure 3, $\left(x_{R X}, y_{R X}\right)$ is location of D2D RX and $\left(x_{T X}, y_{T X}\right)$ is location of D2D TX. $\left(x_{e N B}, y_{e N B}\right)$ is location eNB. $R_{e N B, R X}$ is distance between D2D RX and eNB, $R_{e N B, R X}$ is distance between D2D TX and eNB, and $R_{R X, T X}$ is distance between D2D RX and D2D TX. The eNB know location information of all device. So, it is 
possible to know the distance between the eNB and each device, between one of device and other device. After the eNB calculates the distance between D2D Rx and CUE, the eNB creates a list of cellular network resource that can be shared. The eNB are sort of a list in descending order. this time, it is required that the distance between CUE and D2D Rx is larger than that between CUE and the eNB. After creating a resource list that can be shared, if two different D2D links have a conflict, the D2D pair which is lager distance from CUE has the priority for this resource and other D2D pair exclude the use of this common resource in this allocation. Finally, D2D link shares the resource of CUE from the determined resource list.

\section{The Proposed Scheme}

In this paper, the environment of proposed scheme is considered only the uplink of single cellular network. eNB handle the resource and location management in order to allocate resource efficiently and calculate the distance between device and device and between deivce and eNB. Proposed scheme is composed of two parts. First, eNB select using cellular resource through location information. In the second stage, D2D link allocate the proper resource among selected resources through previous process.

\subsection{CUE Selection for Resource Allocation}

When eNB knows the positions of all UEs, all the distances between CUE and D2D link can be calculated from these informations. When we know the locations of each UEs and $\mathrm{eNB}$, the distance between CUE and one of D2D link, $R_{D 2 D R X, C U E}$, can be determined by

$$
R_{D 2 D R X, C U E}=\sqrt{\left(x_{D 2 D R X}-x_{C U E}\right)^{2}+\left(y_{D 2 D R X}-y_{C U E}\right)^{2}}
$$

where the coordinate of CUE and D2D are referred to as $\left(x_{C U E}, y_{C U E}\right)$ $\left(x_{D 2 D R X}, y_{D 2 D R X}\right)$, respectively. From (5), eNB calculates the values of distance between one D2D link and all the CUEs. Then, eNB sorts the calculated distances between CUE and D2D link in descending order. We select some part of resources from the sorted CUE list since they means the shorter distance between D2D link and CUE uplink and it also indicate that the less interference between them even if they share the resources in the cell.

\subsection{D2D Resource Allocation Algorithm}

After performing the process in Section 3.1, each D2D link can obtain the available lists of CUE resource to be shared. SINR for D2D link is required to consider the interference from CUE as well as the interference from other D2D links which use the same resource with them. The $S I N R_{D 2 D}$ can be determined by

$$
S I N R_{D 2 D}=\frac{P_{D 2 D} G_{D 2 D} \text { air }}{P_{\text {noise }}+\sum_{c=1}^{C_{m a x}} y_{c, d}\left(P_{\text {Cellular }} G_{c, d}+\sum_{k=1}^{D \max } y_{c, k} P_{D 2 D} G_{d, k}\right)}
$$

Where $y_{c, k}$ means that other D2D link k share the resource of the CUE c. $G_{d, k}$ is channel gain between D2D link d to calculate SINR and other D2D link k to use same resource. After calculating SINR of CUE and D2D link from (1) and (6), the maximum throughput rate of CUE and D2D link can be determined by using the equation (3), (4). The maximum throughput is finally shown as

$$
\operatorname{Max}\left(\sum_{k=1}^{d} R_{k}^{D 2 D}+\sum_{m=1}^{c} R_{m}^{\text {Cellular }}\right)
$$

After calculating the maximum throughput rate, D2D link shares the CUE resource as the throughput rate reached the maximum value in (7).

Total process of The proposed scheme is shown Figure 4. 


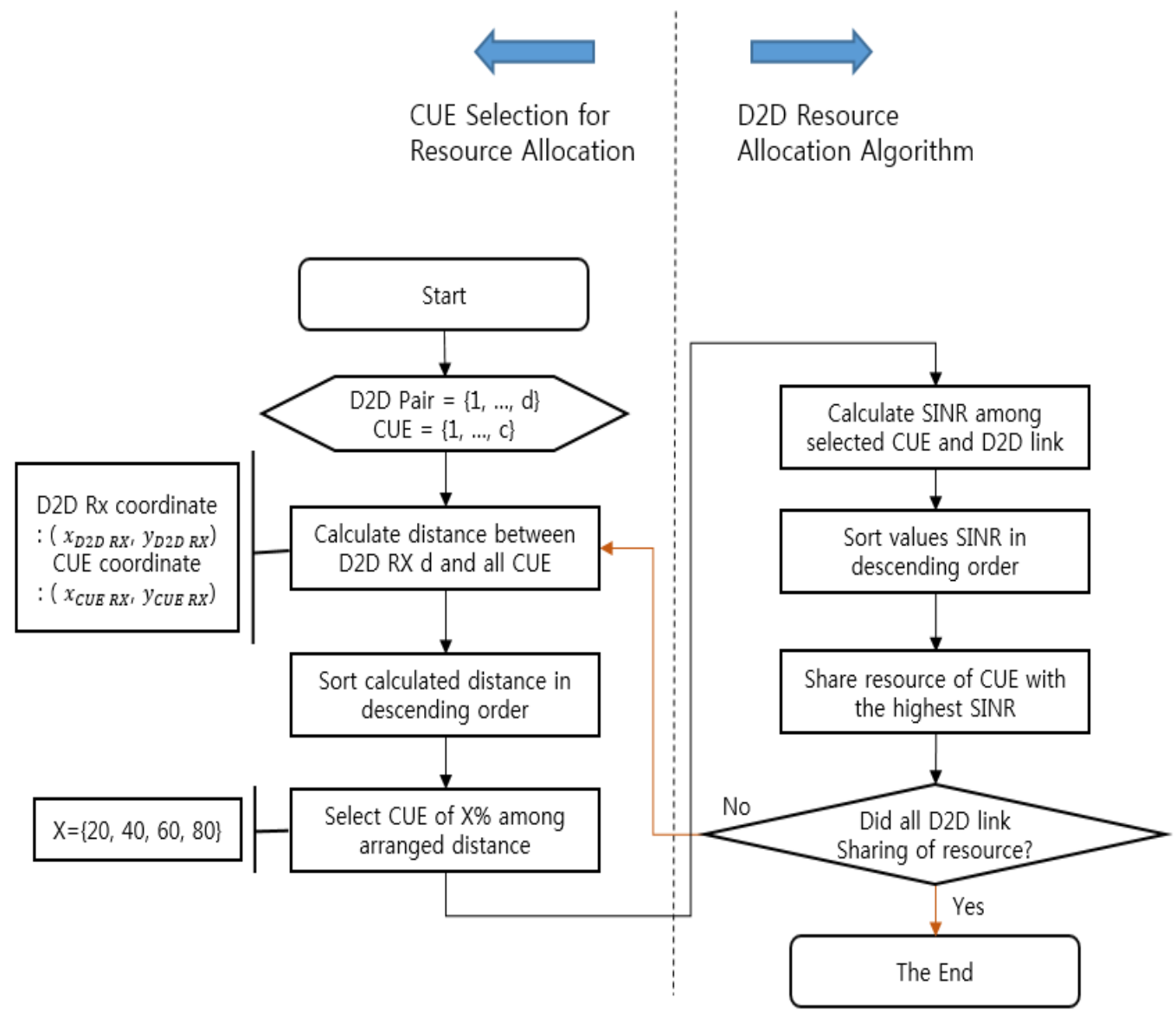

Figure 4. Flow Chart of the Proposed Scheme

\section{Simulation Results}

Table 1. Simulation Environment

\begin{tabular}{|c|c|}
\hline Parameter & value \\
\hline Cell radius & $500 \mathrm{~m}$ \\
\hline Maximum distance between D2D links in a \\
link
\end{tabular}

Simulation environment in this paper is presented in table 1 , the code for simulation is designed by using $\mathrm{C}++$.

Transmission power of cellular users determines to consider with Cell radius and Maximum distance between D2D link and pathloss because this paper don't consider with power control in order to control interference by sharing same resource. 


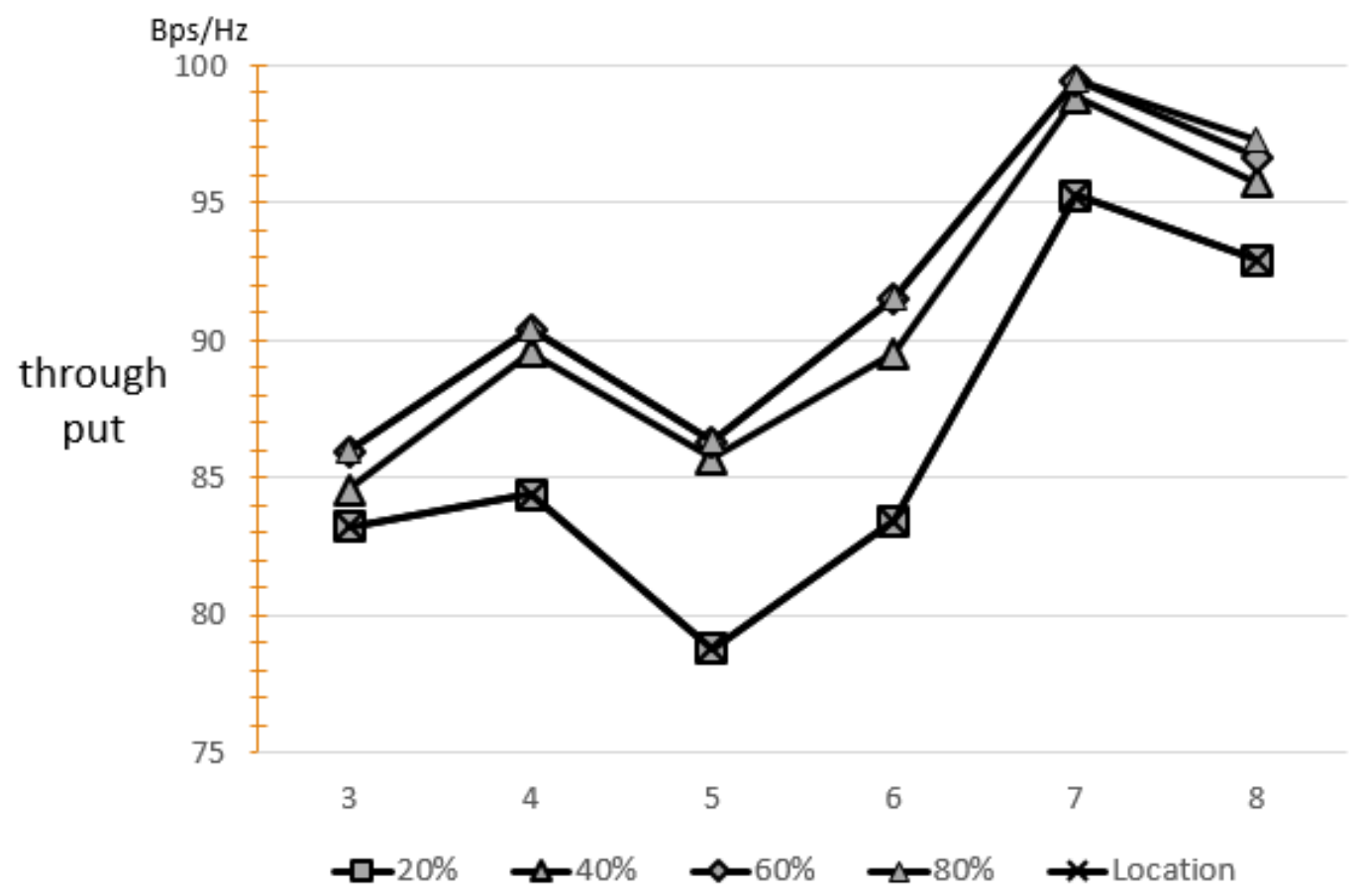

Figure 5. Total Throughput According to Number of D2D Link

Figure 5 shows the total throughput for the four different resource percentage and Location scheme. Location algorithm takes into account only the position information as we already explained in 3.1, and then allocate resource. Proposed scheme uses the top X\% portion of available CUE resources to allocate the resource to D2D link. X has percentage of 20, 40, 60, 80. Environment that shown in Figure 5 is seem with Figure 6 and Figure 7.

The proposed scheme with the exception of $20 \%$ attains the higher throughput over location only scheme. The location only scheme computes to use only distance information without considering the interference on other D2D link and CUE. So, the proposed algorithm utilizes the SINR value after selecting X\% of out of the sorted lists based on the distance from D2D link. It can guarantee the avoidance of selecting resources of occurring the strong interference to D2D link. The proposed scheme with $20 \%$ is the same throughput as location only scheme because top $20 \%$ of all resources have one of resource in simulation environment of this paper. Other percentage is similar to each other percentage. 


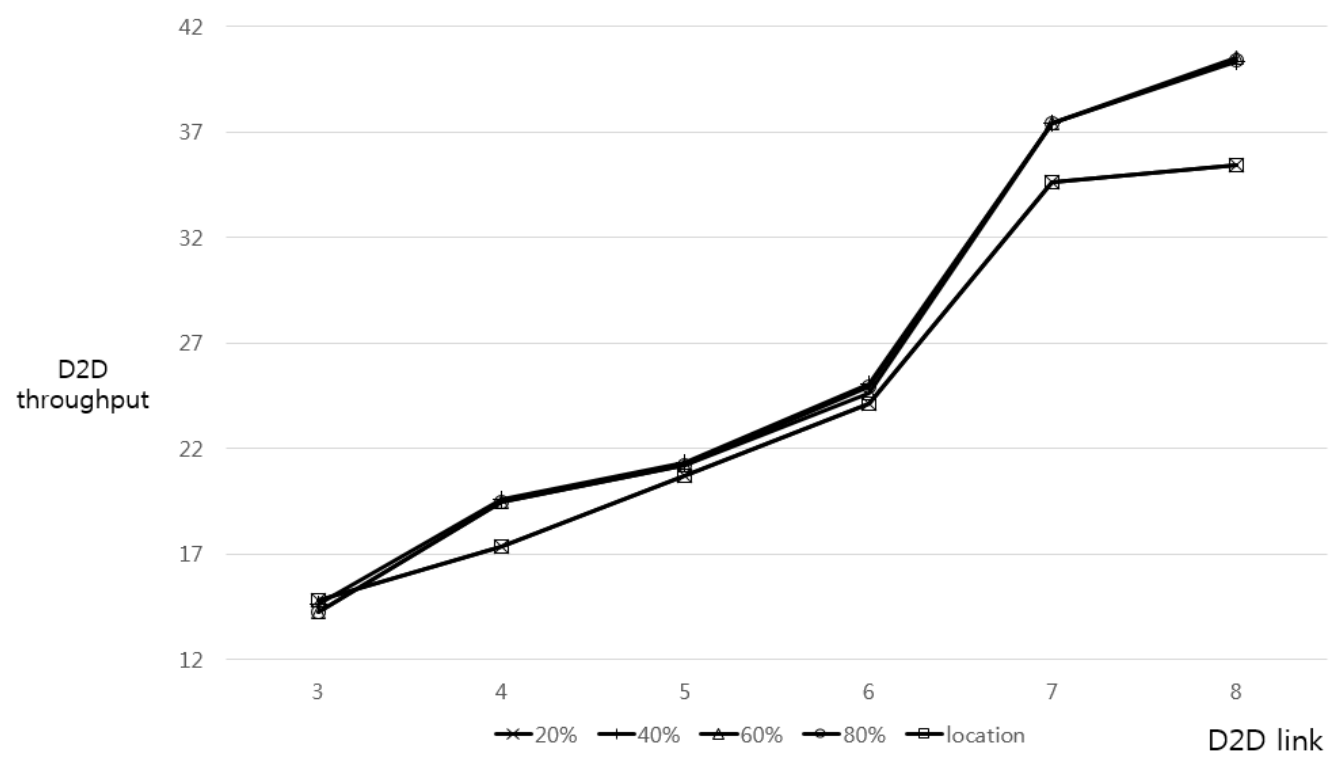

Figure 6. D2D Throughput According to Number of D2D Link

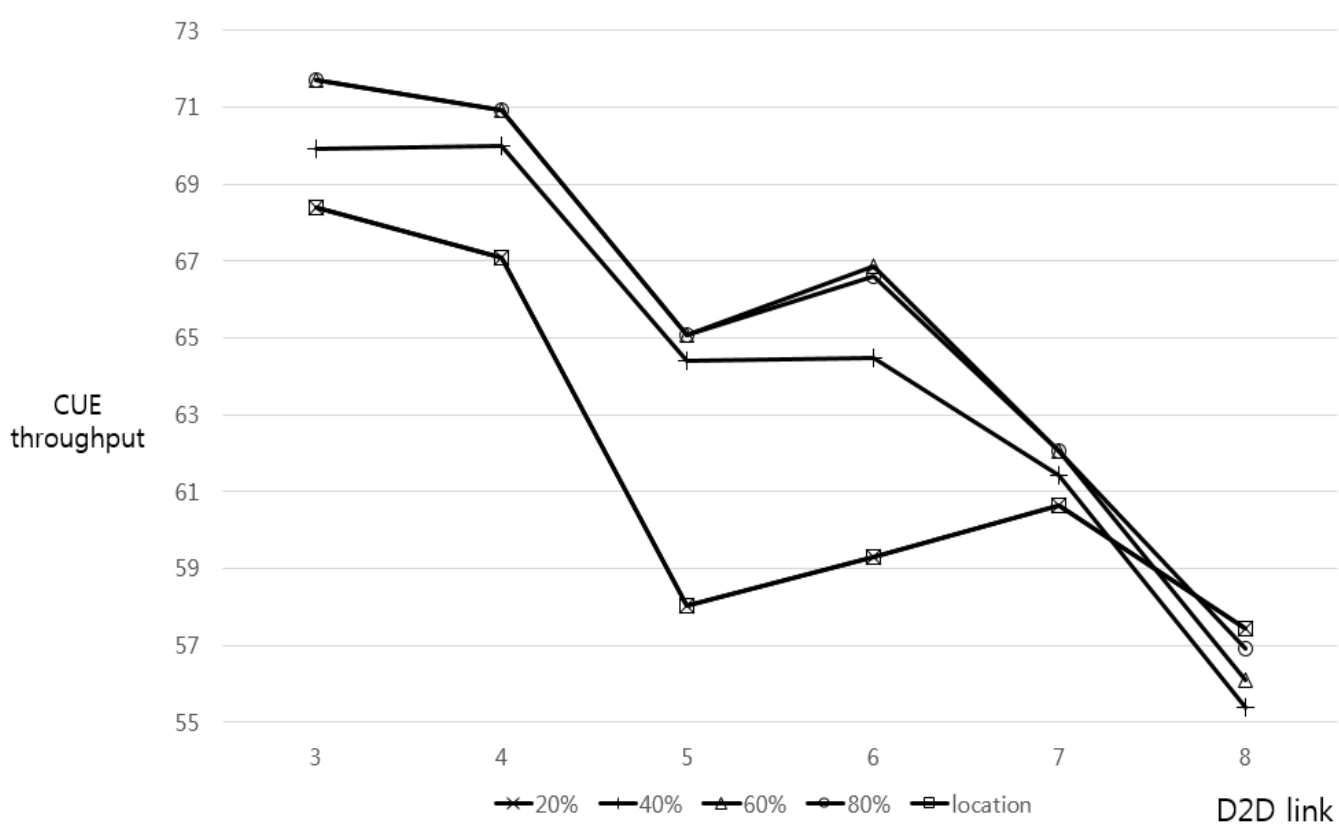

Figure 7. CUE Throughput According to Number of D2D Link

Figure 6 shows the D2D throughput for the four different resource percentage and Location scheme. Graph feature of D2D throughput is similar to total throughput. The proposed scheme with the exception of $20 \%$ attains the higher throughput over location only scheme. The proposed scheme with $20 \%$ is the same throughput as location only scheme. But, graph of D2D throughput increase consistently to be different from graph of total throughput because the proposed scheme ensures D2D link more than cellular user.

Figure 7 shows change of the CUE throughput is severe because the proposed scheme and location only scheme have purpose to ensure mainly D2D link. total throughput is affected by CUE throughput. The proposed scheme ensures better than location only scheme due to calculate maximum throughput along SINR information. But a selected resource by using location information isn't the best choice because of obstacle and interference. 
On the whole, top $40 \%$ of all resources or more are shown that higher than top $20 \%$ and location only scheme. And top $40 \%$ is similar to top $60 \%$ and top $80 \%$. So, we choose 40 percentages in order to select resource when we purpose resource allocation efficiently.

\section{Conclusions}

In this paper, we investigate the resource allocation in the D2D communication based uplink cellular network. The proposed scheme combines the location information with conventional SINR based resource allocation. It selects some portion of resources utilizing location information and share one of selected resource by calculating SINR. Simulation results show that proposed scheme with the exception of $20 \%$ is higher throughout over location only scheme and that selected resource of $40 \%$ among proposed scheme is higher effectiveness over other percentage from the viewpoint of computational complexity and throughput.

In addition, the resource allocation in this paper assumes only the single cell network and consider the interference from CUE and other D2D pairs. Hence, our future works can be the combination or the additional consideration of multicast, power control, data priority, multi cell and QoS driven D2D data transmission.

\section{References}

[1] P. Bhat, "LTE-Advanced: An operator perspective", IEEE Commun. Mag., vol. 50, (2012), pp. 104-114.

[2] M. S. Corson, R. Laroia, J. Li, V. Park, T. Richardson and G. Tsirtsis, "Toward proximity aware internetworking", IEEE Wireless Commun., vol. 17, no. 6, (2010), pp. 26-33.

[3] Y. Xiao, D. Niyato, K.C. Chen and Z. Han, "Enhance Device-to-Device Communication with Social Awareness: A Belief-Based Stable Marriage Game Framework", IEEE Wireless Communications, vol. 23, no. 4, (2016), pp. 36-44.

[4] 3GPP, "Feasibility study for proximity services(ProSe) (Release 12)", TR 22.803, V12.2.0, (2013).

[5] 3GPP, "Work item proposal for enhanced LTE device to device proximity services", RP-142311, (2014)

[6] K. Doppler, M. Rinne, C. Wijting, C. B. Ribeiro and K. Hugl, "Device-to-device communication as an underlay to LTE-advanced networks", IEEE Communications Magazine, vol. 47, no. 12, (2009), pp. 42 49.

[7] C. H. Chien, Y. C. Chen and H. Y. Hsieh, "Exploiting spatial reuse gain through joint mode selection and resource allocation for underlay device-to-device communications", Wireless Personal Multimedia Communications (WPMC), 2012 15th International Symposium on, (2012).

[8] M. Zulhasnine, C. Huang and A. Srinivasan, "Efficient resource allocation for device-to-device communication underlaying LTE network", Wireless and Mobile Computing, Networking and Communications (WiMob), 2010 IEEE 6th International Conference on, (2010).

[9] M. Rodziewicz, "Location-based mode selection and resource allocation in cellular networks with D2D underlay", European Wireless 2015, 21th European Wireless Conference, Proceedings of, (2015).

[10] S. H. Kang and J.G. Kim, "Location Based Resource Allocation in D2D Communication for Cellular Networks", The 10th 2016 International Interdisciplinary Workshop Series, vol. 4, (2016), pp. 87-91.

\section{Authors}

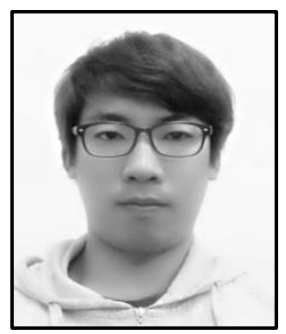

Soo Hyeong Kang, he was born in seoul, Korea on November 20, 1991. He received the B.S degrees in Electronics Engineering Department from Korea Polytechnic University in February 2016. He enrolled the M.S. degrees in Electronics Engineering Department from Korea Polytechnic University in February 2016 until present.

Interests: wireless communication, D2D(Device-to-Device) communication, MIMO. 


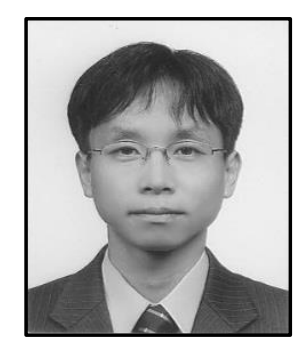

Bangwon Seo, he received his $\mathrm{BS}$, MS, and $\mathrm{PhD}$ degrees in electrical engineering from Korea Advanced Institute of Science and Technology, Daejeon, Rep. of Korea, in 1997, 1999, and 2010, respectively. From November 2004 to February 2013, he was with the Electronics and Telecommunications Research Institute, Department, Rep. of Korea.

In March 2013, he joined the Division of Electrical, Electronic and Control Engineering, Kongju National University, Cheonan, Rep. of Korea, where he is currently an assistant professor. His research interests include device-to-device (D2D) direct communications, OFDM systems, MIMO systems, massive MIMO, CoMP, MCCDMA systems, precoder design, and cognitive radio networks.

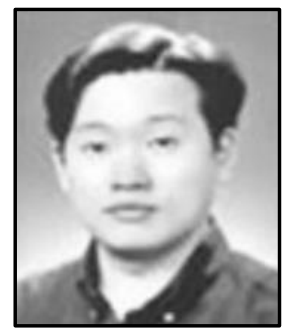

Jeong Gon Kim, he was born in Seoul, Korea on May 24, 1969. $\mathrm{He}$ received the B.S., M.S. and Ph.D, degrees all in electrical engineering from Korea Advanced Institute of Science and Technology (KAIST), Daejeon, Korea in 1991, 1993 and 1998, respectively. From 1998 to 1999, he was the Post-Doctoral Research Fellow at the University of Hawaii at Manoa, USA, From 1999-2001, he joined R\&D center of LG Telecom, Korea and is involved in IMT2000 radio access technology development. From 2001-2003, he was also involved in $3 \mathrm{GPP}$ physical layer standardization, concentrating on the TDD mode in the Telecommunication Research center of Samsung Electronics. Since 2003, he is now a Professor at the Department of Electronics Engineering of Korea Polytechnic University. His research Interests now include the design and performance analysis of wireless communication system, specially 5G mobile communication, MIMO, cooperative communication, and WBAN application. 